\title{
The Virtual Hospitalist: A Single-Site Implementation Bringing Hospitalist Coverage to Critical Access Hospitals
}

\author{
Ethan F. Kuperman, MD, MS*, Eric L. Linson, MBA ${ }^{1}$, Kate Klefstad, MHA², Evelyn Perry, MHA ${ }^{1}$, Kevin Glenn, MD, MS
}

${ }^{1}$ University of lowa Carver College of Medicine, lowa City, lowa; ${ }^{2}$ University of lowa Health Ventures, Coralville, lowa.

BACKGROUND: On-site hospitalist care can improve patient care, but it is economically infeasible for small critical access hospitals (CAHs). A telemedicine "virtual hospitalist" may expand CAH capabilities at a fractional cost of an on-site provider.

OBJECTIVE: To evaluate the impact of a virtual hospitalist on transfers from a CAH to outside hospitals.

DESIGN, SETTING, AND PARTICIPANTS: A six-month pilot program providing "virtual hospitalist" coverage to patients at a CAH in rural lowa.

MEASUREMENTS: The primary outcome was the rate of outside transfers from the CAH Emergency Department (ED). The secondary outcomes included transfer from either the ED or the inpatient wards, daily census, length of stay, transfers after admission, virtual hospitalist time commitment, and patient and staff satisfaction. The preceding 24-week baseline was compared with 24 weeks after implementation, excluding a two-week transition period.

RESULTS: At baseline, there were 947 ED visits and 176 combined inpatient and observation encounters, compared to 930 and 176 after implementation, respectively. Outside transfers from the ED decreased from $16.6 \%$ to $10.5 \%$ $(157 / 947$ to $98 / 930, P<.001)$, and transfers at any time decreased from $17.3 \%$ to $11.9 \%$ (164/947 to $111 / 930, P$ $<.001)$. Daily census, length of stay, and transfers after admission were unchanged. Time commitment for a virtual hospitalist was 35 minutes per patient per day. The intervention was well received by the CAH staff and patients.

CONCLUSIONS: The virtual hospitalist model increased the percentage of ED patients who could safely receive their care locally. A single virtual hospitalist may be able to cover multiple CAHs simultaneously. Journal of Hospital Medicine 2018;13:759-763. Published online first September 26, 2018. () 2018 Society of Hospital Medicine
T hrough increased involvement with families and caregivers, community hospitals can deliver better healthcare to patients. ${ }^{1,2}$ Furthermore, when patients bypass local hospitals and directly present to tertiary care, mortality for time-sensitive illnesses, such as sepsis, increases. ${ }^{3}$ Unfortunately, although critical access hospitals (CAHs) had an equivalent risk-adjusted mortality in 2002, they have failed to improve their performance at the same rate as that of larger hospitals and lag in quality metrics. ${ }^{4,5}$

One potential contributor to the lagging performance may be the low uptake of the hospitalist model at these facilities. ${ }^{6} \mathrm{Al}$ though dedicated hospitalists have improved patient outcomes and decreased spending in large hospitals, ${ }^{7-9}$ implementing the hospitalist medicine model on a smaller scale remains difficult. Approximately 1,300 CAHs provide necessary emergency de-

Address for correspondence: Ethan F. Kuperman, Clinical Assistant Professor, Department of Internal Medicine, University of lowa Carver College of Medicine, SE 622 GH, 200 Hawkins Drive, lowa City, IA 52242; Telephone: 319-3537053; Fax: 319-356-3086; E-mail: ethan-kuperman@uiowa.edu

Additional Supporting Information may be found in the online version of this article.

Received: February 16, 2018; Revised: May 18, 2018; Accepted: June 1, 2018 (๔ 2018 Society of Hospital Medicine DOI 10.12788/jhm.3061 partment (ED) and inpatient services in the rural United States. ${ }^{10}$ Assuming 12-hour shifts and every-other-week assignments, providing continuous, on-location hospitalist coverage would require more than $10 \%$ of the total hospitalist workforce to cover less than $3 \%$ of all hospital admissions. ${ }^{11-13}$

Telemedicine allows content experts, including hospitalists, to supervise patient care remotely. This provides a potential solution to the logistical challenges of supplying continuous hospitalist coverage to a remote facility with a low daily census. We hypothesized that providing continuous "virtual hospitalist" coverage through telemedicine could increase the ability of a CAH to care for patients locally, decreasing the number of transfers to tertiary care centers and improving patient and provider satisfaction. We aimed to create a $25 \%$ relative reduction in CAH Emergency Department patient encounters resulting in transfer to outside hospitals within six months.

This quality improvement project was exempt from Institutional Review Board review.

\section{METHODS}

\section{Setting}

The University of lowa Hospitals and Clinics (UIHC) is a 750bed teaching hospital based in a suburban community in Eastern lowa and the only tertiary care hospital in the state of lowa. The UIHC Hospitalist group contains 44 staff physicians and 
covers more than 12 service lines (both faculty-only and resident-covered) at this facility.

Van Buren County Hospital (VBCH) is a 24-bed CAH offering emergency, internal medicine, and obstetrical services and located 80 miles southwest of UIHC. X-ray and CT scan services are available continuously, but ultrasound and magnetic resonance imaging services are available only two to three times per week. While tertiary care patients were transferred to UIHC, patients requiring specialty care but with less complex illnesses (eg, stable myocardial infarction) were referred to closer facilities.

Prior to implementation, coverage of the acute inpatient ward and the ED at VBCH was simultaneously provided by a single physician or advanced practice providers (APPs). When APPs provided coverage, a physician was required to be notified of any new admissions and was immediately available for medical emergencies. The $\mathrm{VBCH}$ providers worked alone in 48- to 72-hour continuous shifts as the sole coverage for both ED and inpatient units. It was frequently necessary to bring in outside providers through locum tenens agencies to fill gaps in the provider schedule. Both VBCH and UIHC used a shared electronic medical record (EMR), which was a key consideration in choosing VBCH as our pilot site. Providers at both institutions had access to identical patient information through the EMR, including radiology images, laboratory results, and provider notes.

\section{Intervention Development and Implementation}

A site visit by clinical and administrative project leads to $\mathrm{VBCH}$ identified three deficits that we could address through telemedicine: (1) The extended duration of $\mathrm{VBCH}$ shifts was detrimental to provider experience and retention; (2) Lack of local expertise in hospital medicine led to limited comfort in caring for patients with stable but medically complex conditions (eg, drug-resistant urinary tract infection); and (3) Patient transitions between VBCH and UIHC during acute care transfer were frustrating and led to negative experiences with providers and patients.

We developed a model to address these deficits using the minimum number of specialties and employees to facilitate rapid implementation. Although local care ED and inpatient care was provided by three APPS and a single physician provider, we mandated the coverage of all acute inpatients by the virtual hospitalists. This coverage included daily videoconference patient rounds, continuous pager coverage for new acute issues, and listing the virtual hospitalists as the attending of record for patient admissions. We scheduled contact times in the morning and afternoon to accelerate familiarity and comfort with the technology. We used a secure, Health Insurance Portability and Accountability Act of 1996 (HIPAA)-compliant platform for videoconferencing, accessible through personal computers or portable smart devices (Vidyo, Vidyolnc, Hackensack, New Jersey). At VBCH, two tablet computers were provided to serve as portable platforms to use either in provider conference rooms or to be taken into patient rooms. Twice a day, at 8:45 AM and 4:30 PM, virtual hospitalists, local providers, and nursing staff would videoconference and review the status and care plan for all admitted patients. In addition, virtual hospitalists performed a videoconference interview using the tablet computers with all patients on the morning following admission and at other times on an as-needed basis. We asked the virtual hospitalists to cover a minimum of 72 consecutive hours to maintain provider continuity. Local APPs documented the history, examination, and medical decision-making for billing purposes, which were cosigned by the virtual hospitalists. The virtual hospitalists also created separate notes documenting their discussions with local staff, interview and limited direct physical examination findings (eg, appearance of rashes), and medical decision making. Due to limitations of the EMR, local APPs wrote patient orders. All virtual hospitalists were credentialed by proxy at $\mathrm{VBCH}$. We consulted with the UIHC legal team to ensure that virtual hospitalists would be protected under their existing malpractice insurance.

\section{Outcome Measures}

Outcome measures were divided into three categories: (1) clinical and utilization outcomes; (2) virtual hospitalist outcomes; and (3) satisfaction outcomes. The primary clinical outcome was the percentage of ED encounters resulting in transfer to a different acute care hospital. We also monitored alternative ED dispositions, including local inpatient admission. Additional clinical and utilization outcomes after ED admission included the mean daily inpatient census at $\mathrm{VBCH}$ and the case mix index (CMI). We selected the mean length of stay, the percentage of inpatients transferred to other hospitals, and the inpatient mortality as balance measures due to concerns of increasing the acuity of the inpatient wards beyond the comfort and expertise of local staff. Virtual hospitalist outcomes included the mean daily time commitment and the mean time commitment per patient. Virtual hospitalists self-reported their time commitments as part of their daily documentation. We chose these measures in anticipation of expanding this program to other institutions in the future. Satisfaction outcomes included a weekly survey to all VBCH physicians and nursing staff (Appendix 1), weekly group discussions with virtual hospitalists and $\mathrm{CAH}$ staff, and three interviews with patients and family members after discharge (Appendix 2).

\section{Statistical Analysis}

Baseline data collected over a period of 24 weeks were used to measure pre-implementation performance and trends at $\mathrm{VBCH}$. The virtual hospitalist service was started on November 15,2016 , and the two weeks before and two weeks after this date were excluded from analysis as a transition period. To account for weekend variation, we reported data in consecutive 28-day blocks. We used Chi-square tests to compare proportional outcomes and Student's t-tests for continuous variables. Statistical Process Control charts were used to evaluate for temporal trends in quantitative data.

\section{Funding}

Development of this project was funded through the University of lowa Hospitalist group and the Signal Center for Health Innovations at UI Health Ventures. Virtual hospitalist clinical time 
was paid for by the $\mathrm{CAH}$ on a fractional basis of a traditional hospitalist based on projected patient volumes through analysis of baseline data. Patients were not directly billed for virtual hospitalist service but were charged for the services provided by $\mathrm{CAH}$ providers.

\section{RESULTS}

\section{Clinical and Utilization Outcomes}

During the 24-week baseline period, VBCH had 947 ED encounters and 176 combined acute inpatient and observation admissions. For the 24 weeks following the transition, there were $930 \mathrm{ED}$ visits and 186 admissions. We observed a $36 \%$ $(157 / 947$ to 98/930, $P<.001)$ decrease in ED encounters ending in patient transfer to another hospital (Figure). In parallel, VBCH ED visits leading to local admission increased by $62 \%$ of baseline (39/947 to 62/930, $P=.014$ ). There was no significant change in the fraction of ED encounters resulting in an observation stay (104/947 to 99/930, $P=.814$ ). Daily ED visits did not change after virtual hospitalist coverage began (5.64 to 5.54 visits/day, $P=.734$ ), but the percentage of $E D$ visits ending in discharge to a nonmedical setting increased from $79.0 \%$ to $82.7 \%$ (748/947 to $769 / 930, P=.042)$.

The implementation did not have a significant impact on ward census or patient complexity (Table 1). Both CMI and mean length of stay did not change after starting the service. The study was underpowered to detect differences in rare events, including inpatient mortality and transfer after admission. Despite the decrease in transfers, inpatient census was unchanged. This coincides with a 17\% decrease (196/947 to $160 / 930, P=.054$ ) in the proportion of ED patients referred for admission either locally or at an outside hospital.

\section{Virtual Hospitalist Outcomes}

The commitment required for virtual hospitalist responsibilities varied but remained compatible with additional local service, including supervising house staff. When supervising residents, virtual hospitalist responsibilities were performed during resident prerounds and after staffing afternoon consults. Virtual hospital-

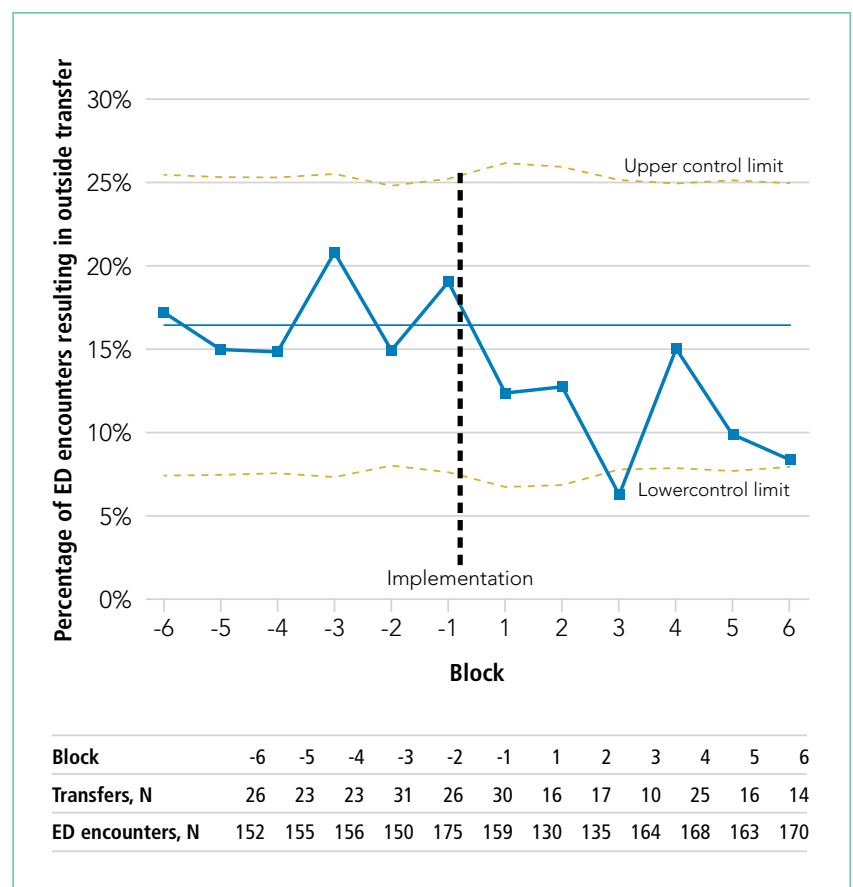

FIG. P-chart of the percentage of ED visits resulting in transfer to an outside hospital. The intervention occurred between blocks -1 and 1 (dotted line). Dashed lines represent upper and lower control limits. After implementing the virtual hospitalist service, the percentage decreased from $16.6 \%$ to $10.5 \%$ (157/947 to 98/930, $P<.001)$.

Abbreviation: ED, emergency department.

ists reported a mean time commitment of 35 minutes per patient per day and 92 total minutes per day on a combination of reviewing and entering data into the EMR, conferencing with VBCH staff, and telemedicine patient encounters. Virtual hospitalists reported spending two or more hours on 31 of 144 shifts.

\section{Satisfaction Outcomes}

The staff at VBCH identified several benefits to the virtual hospitalist service. Survey responses $(N=18)$ were positive, with staff expressing specific gratitude for the additional education

TABLE 1. Characteristics of Patients at the Critical Access Hospital before and after the Virtual Hospitalist Program

\begin{tabular}{|c|c|c|c|}
\hline & Preintervention & Postintervention & $P$ Value \\
\hline \multicolumn{4}{|l|}{ Mean daily census, patients/day (N) } \\
\hline Acute inpatient & $1.30(72)$ & $1.45(87)$ & .511 \\
\hline Observation & $0.69(104)$ & $0.68(99)$ & .981 \\
\hline Combined & $1.98(176)$ & $2.13(186)$ & .513 \\
\hline \multicolumn{4}{|l|}{ Mean length of stay, days (N) } \\
\hline Acute inpatient & $3.13(72)$ & $2.77(87)$ & .237 \\
\hline Observation & $1.11(104)$ & $1.15(99)$ & .592 \\
\hline Combined & $1.92(176)$ & $1.94(186)$ & .896 \\
\hline Case mix index (N) & $0.945(139)$ & $0.955(119)$ & .782 \\
\hline ED encounters transferred before acute discharge, \% (N) & $17.3(164)$ & $11.9(111)$ & $<.001$ \\
\hline Acute inpatients transferred, \% (N) & $9.7(7)$ & $16.1(13)$ & .238 \\
\hline Inpatient mortality, \% (N) & $4.17(3)$ & $1.15(1)$ & .227 \\
\hline
\end{tabular}


TABLE 2. Selected Critical Access Hospital Staff and Patient Comments

"Everything we felt was wonderful."
"Our eyes were opened to how much patient care could be enhanced by this project."
"After the first night, [nurse practitioner] was more excited than when we started."
"[I have] never been so proud to be a part of [our hospital]...[There has] never been anything here [that] has impacted us so quickly and affected level of care
for patients like this."
"I'm very excited for this program, in week one we have learned so much. All staff is wonderful to work with!"
"[The Virtual Hospitalist] is super to work with."

Patient comments

When presented with option to transfer or stay in local hospital: "I am fine here, knowing the University is checking on us."

After transfer: "The doctors worked together as a team to provide the care that I needed. I love that the hospitalist had been following my case from the very beginning. I didn't have to explain everything again when I transferred to [the university hospital] because [the Virtual Hospitalist] already knew the whole story."

and training provided by the virtual hospitalists. On a Likert scale ranging from 1 (very poor) to 5 (excellent), the respondents gave high mean scores to the overall service experience (4.8) and the effectiveness of care delivered (4.9) but were more critical of the ability to keep patients locally (4.5) and the experience with transferring patients (3.9). We also collected freetext feedback from both patients and staff at VBCH (Table 2).

\section{DISCUSSION}

The virtual hospitalist service allowed a higher percentage of acute inpatients to receive care in their local hospital and was positively perceived by providers and patients. The per-patient time commitment by virtual hospitalists was similar to traditional hospitalist coverage ${ }^{14}$ and could scale to multiple simultaneous institutions.

Despite the increase in the proportion of patients admitted locally, neither the mean inpatient census nor the complexity of patients (as measured by CMI) increased. The increase in patients admitted locally was offset by a parallel increase in the number of ED patients discharged home. Although virtual hospitalists were available to consult on ED patients, this consultation was not mandatory unless the $\mathrm{CAH}$ provider felt that admission was indicated. It remains unclear whether the changes in ED disposition were due to direct intervention by virtual hospitalists, increasing local expertise with inpatient medicine, or unrelated local factors.

Although outside transfers directly from the ED dropped, there was a potential increase in acute inpatients transferred after admission that failed to reach statistical significance. We anticipated increased transfers after admission as a potential consequence of accepting more complex patients for $\mathrm{CAH}$ admission. Reasons for transfer included emergent transfers for medically unstable patients and scheduled transfer for subspecialist evaluation or testing. Despite the possible increase in delayed transfers, there was no significant change in $\mathrm{CAH}$ inpatient mortality, and the total fraction of combined ED and inpatients transferred decreased after the intervention.

Despite the benefits of keeping patients within their communities, $20 \%-60 \%$ of rural patients bypass their local facilities when seeking emergent care. ${ }^{15}$ Despite publicity on local media, ${ }^{16}$ we did not observe an increase in daily ED visits after implementation. Although some investigators have found that increasing the services offered decreases in rural bypass, ${ }^{17}$ others have found no or mixed effects. ${ }^{18,19}$ Further investigations into the local factors contributing to rural bypass may yield important insights, and future implementations should not rely on rapid increases in patient volume to establish economic viability.

Although telemedicine has been applied to a variety of previous settings, to our knowledge, this marks the first collaboration between an academic medical center and a $\mathrm{CAH}$ to provide continuous hospitalist coverage. A previous model for pediatric inpatients showed a similar decrease in patients transferred to tertiary centers. ${ }^{20}$ Virtual hospitalists differ from other adult telemedicine projects, which focused on subspecialty care or overnight coverage. ${ }^{21}$ The advantages of our model include the ability to proactively address deficits, even when local providers are unaware of changes to the standards of care. We believe that mandatory scheduled interactions decreased the barriers to communication and increased provider reassurance in telemedicine management of their patients. The scheduled interactions also provided additional training and development for CAH personnel, were well received by local staff, and may contribute to local provider job satisfaction, retention, and recruitment.

Past efforts to integrate academic hospitalists into $\mathrm{CAHs}$ improved quality metrics and provider satisfaction but were economically infeasible due to low patient volumes. ${ }^{22}$ In contrast, virtual providers can distribute their efforts across multiple areas, including covering additional CAHs, providing local patient care at their home facility, or completing academic projects. By combining two or more CAHs into a single provider, sufficient patient volume can be generated to dedicated personnel.

There were several limitations to this initial investigation:

- As a pilot between two specific institutions, modifications will be required to replicate in other $\mathrm{CAHs}$ or academic centers.

- Generating sufficient revenue to cover a full hospitalist salary will require adding additional responsibilities, either covering multiple CAHs simultaneously or combining virtual coverage with in-person responsibilities.

- The accuracy of the self-report remains unmeasured, and the impact of combining two or more $\mathrm{CAHs}$ may not be strictly additive. Attempts to supplement the self-reported time spent with additional information from the EMR and 
cell phone logs were complicated by the use of multiple platforms in parallel, interruptions in provider workflow, and provider multitasking.

- Due to the need for reliable local physical examinations and regulations on telehealth reimbursement, local APPs were necessary for this implementation. Although most of the CAHs have an on-site provider to provide ED coverage, $\mathrm{CAHs}$ with sufficient volume to necessitate separating ED and inpatient ward coverage may have difficulty supporting both APP and virtual hospitalist coverage, even on a fractional basis.

- This study was underpowered to detect rare events with significant consequences, including inpatient mortality and inpatient transfer. Although CMI suggests similar complexity in $\mathrm{CAH}$ patients, we have insufficient data to draw further comparisons on patient characteristics before and after the intervention.

- The analysis may be vulnerable to secular trends in the CAH patient population, as only 24 weeks of data were used as a baseline for comparison (although no significant seasonal variation was detected during that time). Extending the baseline data to include an additional 30 weeks ED encounters did not significantly alter our conclusions.

- Virtual hospitalists were dependent on physical examinations performed independently by local APPs.

- Although virtual providers were obligated to be available for videoconferencing within 60 minutes, more urgent medical decisions were sometimes made based on phone conferences between VBCH and the virtual hospitalist without video or direct patient assessment.

- We selected a CAH utilizing an identical instance of our EMR. Although this increased the ability of virtual hospitalists to split their time between virtual and local patient encounters, this limits our ability to spread this intervention beyond institutions already partnering with the UIHC.

\section{CONCLUSIONS}

We succeeded in reducing outside transfers at a CAH by implementing a sustainable virtual hospitalist service. This model allows patients to receive more of their care within their local communities and provides an improved inpatient experience. Next steps include expanding this service to other CAHs within our region, both to understand if this model is applicable beyond our initial site and to monitor for complications induced by scaling. If successful, virtual hospitalist coverage can provide a sustainable solution to providing the latest innovations in hospital medicine even to the most rural communities.

\section{Acknowledgments}

The authors thank Ray Brownsworth, CEO of Van Buren County Hospital, as well as all the providers and staff who worked with them to implement and improve their services. The authors also thank Pat Brophy, founder of The Signal Center for Health Innovation, for providing leadership, support, and resources for innovation.

Disclosures: None of the authors have identified a conflict of interest in relation to this manuscript.
Funding:This project was funded through the University of lowa Health Care and the Signal Center for Health Innovations at UI Health Ventures.

Compliance with ethical standards: This quality improvement project was exempt from Institutional Review Board review.

\section{References}

1. Kripalani S, Jackson AT, Schnipper JL, Coleman EA. Promoting effective transitions of care at hospital discharge: a review of key issues for hospitalists. $J$ Hosp Med. 2007;2(5):314-323. doi: 10.1002/jhm.228.

2. Potter AJ, Ward MM, Natafgi N, et al. Perceptions of the benefits of telemedicine in rural communities. Perspect Health Inform Manag. 2016;Summer:1-13.

3. Mohr NM, Harland KK, Shane DM, et al. Rural patients with severe sepsis or septic shock who bypass rural hospitals have increased mortality: an instrumental variables approach. Crit Care Med. 2017;45(1):85-93. doi: 10.1097/ CCM.0000000000002026.

4. Joynt KE, Orav EJ, Jha AK. Mortality rates for medicare beneficiaries admitted to critical access and non-critical access hospitals, 2002-2010. JAMA. 2013;309(13):1379-1387. doi: 10.1001/jama.2013.2366.

5. Joynt KE, Harris Y, Orav EJ, Jha AK. Quality of care and patient outcomes in critical access rural hospitals. JAMA. 2011;306(1):45-52. doi: 10.1001/ jama.2011.902.

6. Association AH. AHA Annual Survey Database. Washington, DC: American Hospital Association; 2005.

7. Wachter RM, Katz P, Showstack J, Bindman AB, Goldman L. Reorganizing an academic medical service: impact on cost, quality, patient satisfaction, and education. JAMA. 1998;279(19):1560-1565. doi: 10.1001/jama.279.19.1560.

8. Peterson MC. A systematic review of outcomes and quality measures in adult patients cared for by hospitalists vs nonhospitalists. Mayo Clin Proc. 2009;84(3):248-254. doi: 10.1016/S0025-6196(11)61142-7.

9. Auerbach $A D$, Wachter RM, Katz $P$, et al. Implementation of a voluntary hospitalist service at a community teaching hospital: improved clinical efficiency and patient outcomes. Ann Intern Med. 2002;137(11):859-865. doi: 10.7326/0003-4819-137-11-200212030-00006

10. Moscovice I, Coburn A, Holmes M, et al. Flex Monitoring Team. http://www. flexmonitoring.org/. Accessed December 19, 2016

11. In Critical Condition the Fragile State of Critical Access Hospitals; 2013. http://www.aha.org/research/policy/infographics/pdf/info-cah.pdf. Accessed March 23, 2017.

12. Wachter RM, Goldman L. Zero to 50,000 - the 20th anniversary of the hospitalist. N Engl J Med. 2016;375(11):1009-1011. doi: 10.1056/NEJMp1607958.

13. Aj W, AE. Overview of Hospital Stays in the United States; 2012. http:// www.hcup-us.ahrq.gov/reports/statbriefs/sb180-Hospitalizations-United-States-2012.pdf. Accessed February 7, 2017.

14. Tipping MD, Forth VE, O'Leary KJ, et al. Where did the day go?-A timemotion study of hospitalists. J Hosp Med. 2010;5(6):323-328. doi: 10.1002/ jhm.790.

15. Liu JJ, Bellamy GR, McCormick M. Patient bypass behavior and critical access hospitals: implications for patient retention. J Rural Health. 2007;23(1):17-24 doi: http://dx.doi.org/10.1111/j.1748-0361.2006.00063.x.

16. Keenan $C$. lowa's rural hospitals balance tight budgets with patient needs. The Gazette. July 10, 2017.

17. Escarce JJ, Kapur K. Do patients bypass rural hospitals? Determinants of inpatient hospital choice in rural California. J Health Care Poor Underserved. 2009;20(3):625-644. doi: 10.1353/hpu.0.0178.

18. Liu JJ, Bellamy G, Barnet B, Weng S. Bypass of local primary care in rural counties: effect of patient and community characteristics. Ann Fam Med. 2008;6(2):124-130. doi: 10.1370/afm.794.

19. Weigel PAM, Ullrich F, Ward MM. Rural bypass of critical access hospitals in lowa: do visiting surgical specialists make a difference? J Rural Health. 2018;34 Supplement 1:s21-s29. doi: 10.1111/jrh.12220.

20. LaBarbera JM, Ellenby MS, Bouressa P, et al. The impact of telemedicine intensivist support and a pediatric hospitalist program on a community hospital. Telemed J E Health. 2013;19(10):760-766. doi: 10.1089/tmj.2012.0303.

21. AlDossary S, Martin-Khan MG, Bradford NK, Smith AC. A systematic review of the methodologies used to evaluate telemedicine service initiatives in hospital facilities. Int J Med Inform. 2017;97:171-194. doi: 10.1016/j.ijmedinf.2016.10.012.

22. Dougan BM, Montori VM, Carlson KW. Implementing a Hospitalist Program in a Critical Access Hospital. J Rural Health. 2018;34(1):109-115. doi: 10.1111/ jrh. 12190 